Pacific Journal of Mathematics

THE INDEX OF CONVEXITY AND THE VISIBILITY Gerald A. Be 


\section{THE INDEX OF CONVEXITY AND THE VISIBILITY FUNCTION}

\section{GERALD BEER}

If the integral of the visibility function for a set $E$ is normalized, one arrives at the Index of convexity of $E$, a measure of the relative convexity of $E$ in terms of the average "area seen" by a variable point of $E$. As the visibility function is upper semicontinuous on a compact set in $E_{n}$, the Index is upper semicontinuous on the class of all compact sets in $E_{n}$ with an appropriate metric. We also investigate natural generalizations of convex and starshaped sets in terms of the visibility function.

Definition. The visibility function assigns to each point $x$ of a fixed measurable set $E$ in a Euclidean space $E_{n}$ the Lebesgue outer measure of $S(x)$, the set $\{y: r x+(1-r) y \in E$ for each $r$ in $[0,1]\}$ and zero to each point of $E_{n} / E$.

The study of the visibility function is the study of the dynamic properties of the "star" of a variable point $x$ of $E$; in effect, the techniques used may be described as "starshaped analysis."

If $E$ has positive finite Lebesgue measure, a natural representation of the relative convexity of $E$ is the following Index of convexity:

$$
I(E)=\int_{E} \frac{m(S(x))}{m(E)^{2}} d m(x)
$$

providing this expression makes sense. We show that the Index is upper semicontinuous on the family of all compact sets in $E_{n}$ with an appropriate metric. Finally, we investigate the natural generalizations of convex and starshaped sets in terms of the visibility function and establish desired decomposition theorems for these objects in the compact case.

2. Preliminaries. We essentially use the same terminology as in [3]. We denote ordinary Lebesgue measure in $E_{n}$ by either $m$ or $m_{n}$ (if more than one measure is under discussion). $B_{r}(x)$ will denote the closed $r$-ball about a point $x$; conv ker $E$ and conv $E$ will denote the convex kernel of $E$ and convex hull of $E$, respectively. The interior of a set $E$ relative to the smallest flat containing $E$ is given by intv $E$. Finally, $x y$ will denote the line segment joining $x$ to $y$ and $L(x, y)$ will denote the line determined by $x$ and $y$. We begin with this obvious fact: If $E$ is a closed (open) set in $E_{n}$, then $S(x)$ is closed (open) for each $x$ in $E$. Let us designate the visibility 
function for a fixed set by the letter $v$.

\section{Continuity properties of the visibility function.}

THEOREM 1. If $O \subset E_{n}$ is open, then the visibility function associated with $O$ is lower semicontinuous.

Proof. Let $x \in O$ and let $\left\{x_{n}\right\} \rightarrow x$. Clearly $S(x) \subset \bigcup_{k=1}^{\infty} \bigcap_{n=k}^{\infty} S\left(x_{n}\right)$, for if $p \in S(x)$, there exists a neighborhood about $x$ which sees $p$ via $O$. Hence $v(x)=m(S(x)) \leqq m\left(\bigcup_{k=1}^{\infty} \bigcap_{n=k}^{\infty} S\left(x_{n}\right)\right)=\lim _{k \rightarrow \infty} m\left(\bigcap_{n=k}^{\infty} S\left(x_{n}\right)\right) \leqq$ $\underline{\lim }_{k \rightarrow \infty} m\left(S\left(x_{k}\right)\right)=\underline{\lim }_{k \rightarrow \infty} v\left(x_{k}\right)$. Since $v(x)=0$ when $x \notin O$ and $O$ is open, $v$ is lower semicontinuous.

THEOREM 2. Let $K \subset E_{n}$ be compact. Then the visibility function associated with $K$ is upper semicontinuous.

Proof. Again let $x \in K$ and $\left\{x_{n}\right\} \rightarrow x$. The compactness of $K$ implies that $S(x) \supset \bigcap_{k=1}^{\infty} \bigcup_{n=k}^{\infty} S\left(x_{n}\right)$. We have $v(x)=m(S(x)) \geqq$ $m\left(\bigcap_{k=1}^{\infty} \bigcup_{n=k}^{\infty} S\left(x_{n}\right)\right)=\lim _{k \rightarrow \infty} m\left(\bigcup_{n=k}^{\infty} S\left(x_{n}\right)\right) \geqq \varlimsup_{k \rightarrow \infty} m\left(S\left(x_{k}\right)\right)$. The result now follows as before.

COROLLARY. The visibility function for a compact set when restricted to the set attains a maximum.

COROLlary. The visibility function for a closed set is a Borel function.

Proof. Let $E$ be closed and $x \in E$ be arbitrary. Then $S(x)=$ $\mathrm{U}_{n=1}^{\infty} S(x) \cap B_{n}(0)$ so that $v$ is the limit of upper semicontinuous functions.

ExAmple. A closed set $E$ on which $v$ is not upper semicontinuous. Let $E$ be the following set in the plane:

$$
(0,1) \cup \operatorname{cl} \bigcup_{n=1}^{\infty} \operatorname{conv}((1 / n, 1) \cup\{(r, 0): r \geqq n\}) .
$$

Then $v(0,1)=0$ but $v(1 / n, 1)=\infty$ for all $n$.

Definition. Let $K$ be a compact set in $E_{n}$. The $\varepsilon$-parallel set of $K$, denoted by $K_{\varepsilon}$, is the compact set $\bigcup_{x \in K} B_{\varepsilon}(x)$.

Let $S_{n-1}$ denote the unit sphere in $E_{n}$. Every $x$ in $E_{n} /\{0\}$ can be written uniquely as $r \cdot \theta$ where $r>0$ and $\theta \in S_{n-1}$. If $\sigma_{n-1}$ denotes the standard surface measure for $S_{n-1}$ and if $f$ is a positive Borel 
function on $E_{n}$, recall that

$$
\int_{E_{n}} f d m_{n}=\int_{0}^{\infty} \int_{S_{n-1}} r^{n-1} f(r \cdot \theta) d \sigma_{n-1}(\theta) d r
$$

LEMma. Let $f$ be a nonnegative Borel function defined on $S_{n-1}$. If $x_{0}=f(\theta) \cdot \theta$ for each $\theta \in S_{n-1}$, then $\bigcup_{\theta} x_{\theta}$ is a set of $n$-dimensional measure zero.

Proof. By Lusin's Theorem, $\forall n$ there exists a continuous function $f_{n}$ on $S_{n-1}$ such that $f_{n}=f$ on a compact set whose surface measure differs from that of the unit sphere by less than $1 / n$. Hence $E=$ $\mathbf{U}_{n=1}^{\infty}\left\{x_{\theta}: f(\theta)=f_{n}(\theta)\right\}$ is an $F_{o}$-set differing from $\bigcup_{\theta} x_{\theta}$ by a set of measure zero so that $\mathrm{U}_{\theta} x_{\theta}$ is measurable. Since the characteristic function of $E$ is Borel we have

$$
m\left(\mathbf{U}_{\theta} x_{\theta}\right)=m(E)=\int_{S_{n-1}} \int_{0}^{\infty} r^{n-1} \chi_{E}(r \cdot \theta) d r d \sigma_{n-1}(\theta)=0
$$

as each ray emanating from the origin intersects $E$ in at most one point.

The following corollary is indispensable in studying the visibility function.

Corollary. Let $E$ be a compact set in $E_{n}$. If $x \in E$, the set of endpoints of all segments in $S(x)$ with one end point $x$ forms a measurable set and has measure zero.

Proof. We may assume $x=0$. Let $p_{\theta}$ denote the endpoint in the $\theta$ direction (which may be 0 ). If we apply the previous lemma to the upper semicontinuous function $f(\theta)=\left|p_{\theta}-0\right|$, the result follows.

4. The index of convexity.

Definition. Let $E \subset E_{n}$ be a measurable set with measurable visibility function $v$, and suppose $m(E)<\infty$. If $m(E)>0$, the Index of convexity of $E, I(E)$, is given by

$$
\int_{E} \frac{v}{m(E)^{2}} d m
$$

If $m(E)=0$, we agree to let $I(E)$ be 1 .

Clearly $0 \leqq I(E) \leqq 1$ for any set $E$ for which the Index makes sense, and if $E$ has a nonempty interior, the Index is nonzero. It is evident that the class of sets of Index 1 is closed under countable 
intersections and contains the bounded convex sets as a subclass.

THEOREM 3. Suppose $F_{1} \subset E_{n}$ and $F_{2} \subset E_{l}$ are two sets of positive measure for which the Index is defined. Then $I\left(F_{1} \times F_{2}\right)=I\left(F_{1}\right) I\left(F_{2}\right)$.

Proof. Let $v$ be the visibility function for $F_{1} \times F_{2}$. Clearly $S(x) \times S(y)=S(x, y)$ for every $(x, y) \in F_{1} \times F_{2}$ so that $v(x, y)=v_{1}(x) v_{2}(y)$, where $v_{i}$ is the visibility function for $F_{i}, i=1,2$. The result now follows in the obvious way using Fubini's Theorem.

From the results of Theorems 1 and 2, it is immediate that the Index makes sense for compact and bounded open sets.

If $\left\{E^{n}\right\}$ is a sequence of compact sets which converge to a compact set $E$ in the Hausdorff metric, we might expect some relationship between $\left\{I\left(E^{n}\right)\right\}$ and $I(E)$. However, we are working in the wrong metric space as the two following planar examples show.

(1) Let $E=\{z:|z| \leqq 1\} \cup\{z:|z-2| \leqq 1\}$. Let $E^{n}=\{z:|z-2| \leqq$ $1\} \cup\{z:|z| \leqq 1, \operatorname{Im} z=k / n$ for some integer $k\}$. Then $E^{n} \rightarrow E, I\left(E^{n}\right)=1$ for every $n$, but $I(E)=1 / 2$.

(2) Let $E=\{z: 0 \leqq \operatorname{Im} z \leqq 1,|\operatorname{Re} z| \leqq 1\}$ and let $E^{n}=\{z: 0 \leqq$ $\operatorname{Im} z \leqq 1,1 / n \leqq|\operatorname{Re} z| \leqq 1\}$. Then $E^{n} \rightarrow E, I\left(E^{n}\right)=1 / 2$ for every $n$, but $I(E)=1$.

What goes wrong is our improper generalization of convergence for convex sets, for if convex sets converge in the Hausdorff metric, they also converge in measure. We define a new metric $\bar{d}$ on the class of compact sets in $n$-space. If $A$ and $B$ are two such sets, define $\bar{d}(A, B)=\sup \{d(A, B), m(A \Delta B)\}$ where $d$ denotes Hausdorff distance.

Although this metric space is not complete, we can still generalize that part of Blaschke's theorem which says that the limit of convex sets is convex.

THEOREM 4. The Index of convexity is upper semicontinuous on the metric space $\bar{d}$ of compact sets in $n$-space.

Proof. We show that if $K_{n}$ are compact and converge to $K(\bar{d})$, then $I(K) \geqq \lim \sup I\left(K_{n}\right)$. We may assume $m(K)>0$ by our convention that $I(K)=1$ if $m(K)=0$.

Let $K^{\prime}=\left\{x \in K: x \in K_{n}\right.$ for infinitely many $\left.n\right\}$. We claim $m\left(K^{\prime}\right)=$ $m(K)$. We must show $m\left\{x: x \in K \cap K_{n}^{c}\right.$ for all but finitely many $\left.n\right\}=0$. Suppose $m\left(\bigcup_{k=1}^{\infty} \bigcap_{n>k} K_{n}^{c} \cap K\right)>\delta>0$. Then there exists $N$ such that $m\left(\bigcap_{n>N} K_{n}^{c} \cap K\right)>\delta / 2$. Hence $m\left(K_{n}^{c} \cap K\right)>\delta / 2$ for $n>N$. But $K_{n}^{c} \cap K \subset\left(K \Delta K_{n}\right)$, a contradiction to $m\left(K \Delta K_{n}\right) \rightarrow 0$.

Let $v$ denote the visibility function for $K$ and let $v_{n}$ denote the 
visibility function for $K_{n}$.

We claim for each $x$ in $K^{\prime}, v(x) \geqq \lim \sup v_{n}(x)$. Let $S_{n}(x)=$ $\left\{y: y x \subset K_{n}\right\}$. We need only show that for every $\varepsilon>0$, there exists $N$ such that $n>N \Rightarrow(S(x))_{\varepsilon} \supset S_{n}(x)$ whenever $x \in K_{n}$. Suppose not. Then there exists a sequence of segments $\left\{y_{n_{l}} x\right\}$ such that for each $l, y_{n_{l}} x \subset K_{n_{l}}$, but $y_{n_{l}}$ is not contained in $(S(x))_{\varepsilon}$. Passing to a subsequence we may assume that $\left\{y_{n_{l}} x\right\}$ converges in the Hausdorff metric to a subset $D \subset S(x)$. Hence if $l$ is sufficiently large $y_{n_{l}} x \subset D_{\varepsilon} \subset(S(x))_{\varepsilon}$, a contradiction.

We now have by Fatou's lemma

$$
\begin{aligned}
I(K) & =\int_{K^{\prime}} \frac{v}{m\left(K^{\prime}\right)^{2}} d m \\
& \geqq \int_{K^{\prime}} \lim \sup \frac{v_{n}}{m\left(K_{n}\right)^{2}} d m \\
& \geqq \lim \sup \int_{K^{\prime}} \frac{v_{n}}{m\left(K_{n}\right)^{2}} d m \\
& =\lim \sup \int_{K^{\prime} \cap K_{n}} \frac{v_{n}}{m\left(K_{n}\right)^{2}} d m+\lim \int_{K_{n^{\prime} / K^{\prime}}} \frac{v_{n}}{m\left(K_{n}\right)^{2}} d m \\
& =\lim \sup I\left(K_{n}\right) .
\end{aligned}
$$

Our second example shows that strict inequality may indeed occur.

CoRollary. If $I\left(K_{n}\right)=1$ for each $n$ and if $K_{n} \rightarrow K$ in the $\bar{d}$ metric, then $I(K)=1$.

5. Pseudo kernels. Sets of Index 1 are the obvious generalization of convex sets in terms of "visibility theory." Analogously, a set $E$ is starshaped in a more general sense if there is a point $x$ of $E$ satisfying $m(E)=m(S(x))$. In this section we classify in the compact case sets of Index 1 and "pseudo starshaped" sets.

Definition. Let $E$ be a measurable set in $E_{n}$. The pseudo kernel of $E$ is the set $\{x \in E: v(x)=m(E)\}$. We denote this set by $P$ ker $E$. $E$ is pseudo starshaped if $P \operatorname{ker} E \neq \varnothing$.

Lemma. If $E \subset E_{n}$ is open, $P$ ker $E$ is a $G_{\dot{o}}$-set. If $K \subset E_{n}$ is compact, $P$ ker $K$ is compact.

Proof. If $E$ is open, $P \operatorname{ker} E=\bigcap_{n=1}^{\infty}\{x \in E: v(x)>m(E)-1 / n\}$. If $K$ is compact and $m(K)=0$ the assertion is obviously true; otherwise, Theorem 2 implies that $\{x \in K: v(x) \geqq m(E)\}$ is compact. 
Lemma. Let $K$ be a compact set in $E_{n}$. If conv ker $K$ has a nonempty interior, then conv ker $K=P$ ker $K$.

Proof. Let $y \in P$ ker $K /$ conv ker $K$. Since $K^{c}$ is open, $y$ must see every point of int conv $(x \cup$ conv ker $K)$ where $x \in K$ is arbitrary. Since $K$ is compact, $x \in S(y)$ for every $x \in K$.

ExAmple. A compact set $K$ in $E_{3}$ satisfying $\operatorname{dim}($ conv ker $K)=2$ but conv ker $K \neq P$ ker $K$. Let $K=\left\{(x, y, z): x^{2}+y^{2}+z^{2} \leqq 1\right\} \cup\{(x$, $\left.y, z): x^{2}+y^{2} \leqq 2, z=0\right\}$.

The following property of the visibility function peculiar to compact sets is particularly illuminating in this section.

THEOREM 5. Let $E$ and $F$ be compact sets in $E_{n}$. If $m(E \Delta F)=0$, then the visibility functions for the two sets are equal.

Proof. We first show the two functions agree on $E \cap F$. W.l.o.g., we may assume 0 to be an arbitrary point of $E \cap F$. Let $S_{F}(0)$ be those points of $F$ which 0 sees via $F$ and let $S_{E}(0)$ be those points of $E 0$ sees via $E$. Suppose $m\left(S_{E}(0)\right)>m\left(S_{F}(0)\right)$ so that certain segments in $S_{E}(0)$ contain segments in $F^{c}$.

Consider the function $f$ defined on $S_{n-1}$ given by $f(\theta)=\sup \{|x-y|$ : $\left.x y \subset F^{c} \cap R_{\theta} \cap S_{E}(0)\right\}$ where $R_{\theta}$ denotes the ray from 0 through $\theta$. We claim $f$ is a Borel function. To see this, note that $f(\theta)=\inf f_{n}(\theta)$ where $f_{n}(\theta)=\sup \left\{|x-y|: x y \subset F^{c} \cap R_{\theta} \cap\left(S_{E}(0)\right)_{1 / n}\right\}$. However, for every $n$ the $1 / n$-parallel set about $S_{E}(0)$ is a compact starshaped set whose convex kernel has a nonempty interior. Hence, the boundary is a continuous function of $\theta$ which implies $f_{n}$ is lower semicontinuous for every $n$, so that $f$ is a Borel function. Now we must have $\sigma_{n-1}\left(f^{-1}(0, \infty)\right)>0$. Hence there exists $\delta>0$ such that $\sigma_{n-1}\{\theta$ : there exists $\left.x y \subset F^{c} \cap R_{\theta} \cap S_{E}(0),|x-y|>\delta\right\}>0$. Since this set, which we denote by $M_{\delta}$, is a Borel set, the set of all points in $F^{c} \cap S_{E}(0)$ which project radially onto $M_{\delta}$ is a Borel set. Denoting this last set by $M$ we have

$$
\begin{aligned}
\int \chi_{M} d m_{n} & =\int_{M \delta_{\delta}} \int_{0}^{\infty} r^{n-1} \chi_{M}(r \cdot \theta) d r d \sigma_{n-1}(\theta) \\
& \geqq \frac{\delta^{n}}{n} \sigma_{n-1}\left(M_{\delta}\right)>0 .
\end{aligned}
$$

But $M \subset S_{E}(0) \cap F^{c} \subset E \cap F^{c}$. Hence we have a contradiction to $m(E \Delta F)=0$. It is easy to show that both visibility functions are zero at any point $x$ in $E \Delta F$ since $E \cap F$ is compact.

Intuitively we should only expect that $I(P \operatorname{ker} K)=1$ and not 
that $P \operatorname{ker} K$ is convex. It is surprising that the following theorem holds.

Theorem 6. Let $K$ by any compact set in $E_{n}$ with $m(K)>0$. Then $P$ ker $K$ is convex.

Proof. Again we may assume that 0 is an arbitrary element of $P$ ker $K$, and let $y$ be any other element of $P$ ker $K$. Since $m(S(0))>0$, every neighborhood of 0 must contain a subset of $K$ of positive measure so that $y$ must see some point in each neighborhood via $K$. The compactness of $K$ implies $y$ sees 0 via $K$.

We claim $0 y \subset P$ ker $K$. Consider the Borel set $S(0) \cap S(y)^{c}$, which has measure zero. We must have for almost every $\theta$ on $S_{n-1}$

$$
\int_{0}^{\infty} r^{n-1} \chi_{s(0) \cap S(y) c}(r \cdot \theta) d r=0 .
$$

Since $R_{\theta} \cap S(0) \cap S(y)^{c}$ is either empty or contains open segments, we have $R_{\theta} \cap S(0) \cap S(y)^{c}=\varnothing$ for almost all $\theta$. This means that $y$ sees every line segment in $S(0)$ in its entirety except a set of lines whose union forms a set of measure zero. Hence any point in intv $0 y$ has the same property. Since almost every point of $K$ is in $S(0)$, $0 y \subset P$ ker $K$.

Corollary. Let $K$ be a compact set in $E_{n}$. Then $I(K)=1$ iff $K=F \cup C$ where $C$ is compact and convex and $m(F)=0$.

COROLlary. (Helly's Theorem for compact sets of Index 1.) Let $\left\{K_{\alpha}\right\}$ be a collection of compact sets of Index 1 in $E_{n}$ such that every $n+1$ intersect in a set of positive measure. Then $\cap K_{\alpha} \neq \varnothing$.

COROLLARY. If $K \subset E_{n}$ is a compact set which is the closure of an open set and $I(K)=1$, then $K$ is convex.

As a consequence of Theorem 5 we have the following

CoROLlaRY. If a compact set $K \subset E_{n}$ satisfies $m(K)>0, I(K)=1$ and $v(x)>0$ for every $x$ in $K$, then $K$ is convex.

We are now in a position to classify those compact sets with nonempty pseudo kernels.

THEOREM 7. Let $K$ be a compact set in $E_{n}$ with $m(K)>0$. Suppose $P \operatorname{ker} K \neq \varnothing$. Then $K=S \cup F$ where $m(F)=0$ and $S$ is a compact starshaped set with convex kernel $P$ ker $K$. In addition, $m(S(x))=0$ for every $x \in F$. 
Proof. Let $\left\{a_{n}\right\}$ be a countable dense subset of $P \operatorname{ker} K$. $m\left(\bigcup_{n=1}^{\infty} K / S\left(a_{n}\right)\right)=0$ so that $m\left(\bigcap_{n=1}^{\infty} S\left(a_{n}\right)\right)=m(K)$. The compactness of $K$ implies

$$
\bigcap_{x \in P \operatorname{ker} K} S(x)=\bigcap_{n=1}^{\infty} S\left(\alpha_{n}\right) \text { so that } m(K)=m\left(\bigcap_{x \in P \operatorname{ker} K} S(x)\right) .
$$

Let $y \in \bigcap_{x \in P \text { kerK } K} S(x)$ and $x_{0} \in P$ ker $K$ be arbitrary. If $q \in \operatorname{intv} x_{0} y$, we have $\operatorname{conv}(q \cup P \operatorname{ker} K) \subset \operatorname{conv}(y \cup P \operatorname{ker} K) \subset K$ so that $q \in \bigcap_{x \in P \operatorname{ker} K} S(x)$. Hence $\bigcap_{x \in P \text { kel } K} S(x)$ is starshaped with respect to $P$ ker $K$, is compact and is of full measure. If $y \in K / \bigcap_{x \in P \text { RerK }} S(x)$, Theorem 5 again implies $v(y)=0$.

We note that any compact pseudo starshaped set has a visibility function identical with the visibility function of some compact starshaped set.

THEOREM 8. Let $\left\{K_{n}\right\}$ be a sequence of compact sets of positive measure with nonempty pseudo kernels $\left\{P_{n}\right\}$ and suppose $\left\{K_{n}\right\} \rightarrow K(\bar{d})$. Then $K$ has a pseudo kernel which contains a subsequential limit of $\left\{P_{n}\right\}$.

Proof. Let $K_{n}=S_{n} \cup F_{n}$ where $S_{n}$ is compact and starshaped with respect to $P_{n}$ and $m\left(F_{n}\right)=0$. By the Blaschke convergence theorem for starshaped sets, passing to a subsequence we may assume $\left\{S_{n}\right\} \rightarrow S$ and $\left\{P_{n}\right\} \rightarrow P$ in the Hausdorff metric, where $P \subset$ conv ker $S$. We have $m(S) \geqq \lim \sup m\left(S_{n}\right)=\lim m\left(K_{n}\right)=m(K)$. Since $S \subset K$, we have $m(K / S)=0$.

Since the compact pseudo starshaped sets include all compact sets of measure zero, it follows from Theorem 8 that they form a closed metric subspace of the metric space of all compact sets under $\bar{d}$. I fails to be continuous on this subspace; to see this, we note that the unit disc in $E_{2}$ is the limit of a sequence of radial Cantor sets of positive measure.

No classification theorem for open sets of Index 1 has yet been established. Clearly, if $E$ is a bounded open set of Index 1, then cl $E$ is convex which implies that a regular bounded open set is convex iff it has Index 1. It seems reasonable to conjecture that the Index of a pseudo kernel of a bounded open set would be 1 . A property shared in common with convex open sets is the following.

THEOREM 9. Let $\left\{E_{m}\right\}$ be a countable collection of bounded open sets in $E_{n}$ of Index 1 such that every $n+1$ contain a translate of some closed ball $B$. Then $\bigcap_{m=1}^{\infty} E_{m} \neq \varnothing$. 
Proof. The collection $\left\{\mathrm{cl}\left(E_{m}\right)\right\}$ satisfy the hypotheses of the following theorem of Klee [1]: If $\left\{K_{\alpha}\right\}$ is a collection of compact convex sets in $E_{n}$ such that the intersection of every subfamily of cardinality $n+1$ contains a translate of some convex compact set $K$, then $\cap K_{\alpha}$ contains a translate of $K$. Therefore, $\bigcap_{m=1}^{\infty} \operatorname{cl}\left(E_{m}\right)$ contains $B+p$ for some $p \in E_{n}$. Now bd $E_{m}$ is nowhere dense in $B+p$ for each $m$. Since $B+p$ is a complete metric space, $\bigcap_{m=1}^{\infty} E_{m}$ is nonempty.

EXAMPLE. For each $\left(r_{1}, r_{2}\right) \in E_{2}$, let

$$
E_{r_{1}, r_{2}}=\left\{(x, y): 0<x<1,0<y<1,(x, y) \neq\left(r_{1}, r_{2}\right)\right\} .
$$

It is easy to see that the intersection of any three such sets contains a ball of radius $1 / 12$, but $\bigcap_{\left(r_{1}, r_{2}\right) \in E_{2}} E_{r_{1}, r_{2}}=\varnothing$.

\section{REFERENCES}

1. V. L. Klee, The critical set of a convex body, Amer. J. Math., 75 (1953), 178-188.

2. Walter Rudin, Real and Complex Analysis, McGraw-Hill, New York, 1966.

3. F. A. Valentine, Convex Sets, McGraw-Hill, New York, 1964.

Received September 14, 1971 and in revised form July 28, 1972.

California State College, Los Angeles 



\section{PACIFIC JOURNAL OF MATHEMATICS}

\section{EDITORS}

H. SAMELSON

Stanford University

Stanford, California 94305

C. R. Hовву

University of Washington

Seattle, Washington 98105
J. DugundJI

Department of Mathematics University of Southern California

Los Angeles, California 90007

RICHARD ARENS

University of California

Los Angeles, California 90024

\section{ASSOCIATE EDITORS}

E. F. BECKENBACH

B. H. NeumanN

F. WOLF

K. YoSHIDA

\section{SUPPORTING INSTITUTIONS}

UNIVERSITY OF BRITISH COLUMBIA

CALIFORNIA INSTITUTE OF TECHNOLOGY

UNIVERSITY OF CALIFORNIA

MONTANA STATE UNIVERSITY

UNIVERSITY OF NEVADA

NEW MEXICO STATE UNIVERSITY

OREGON STATE UNIVERSITY

UNIVERSITY OF OREGON

OSAKA UNIVERSITY
UNIVERSITY OF SOUTHERN CALIFORNIA

STANFORD UNIVERSITY

UNIVERSITY OF TOKYO

UNIVERSITY OF UTAH

WASHINGTON STATE UNIVERSITY

UNIVERSITY OF WASHINGTON

AMERICAN MATHEMATICAL SOCIETY

NAVAL WEAPONS CENTER

Printed in Japan by International Academic Printing Co., Ltd., Tokyo, Japan 


\section{Pacific Journal of Mathematics}

\section{Vol. 44, No. $1 \quad$ May, 1973}

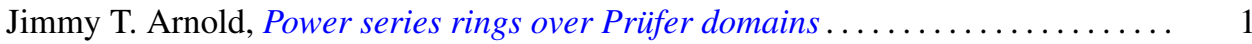

Maynard G. Arsove, On the behavior of Pincherle basis functions . . . . . . . . . 13

Jan William Auer, Fiber integration in smooth bundles ................. 33

George Bachman, Edward Beckenstein and Lawrence Narici, Function algebras

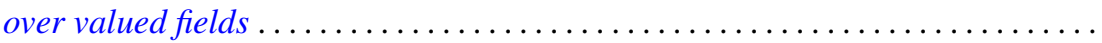

Gerald A. Beer, The index of convexity and the visibility function . . . . . . . . . . .

James Robert Boone, A note on mesocompact and sequentially mesocompact

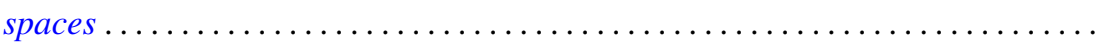

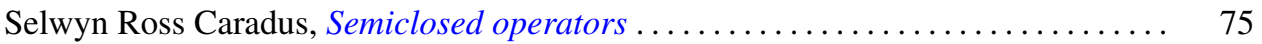

John H. E. Cohn, Two primary factor inequalities . . . . . . . . . . . . . . . 81

Mani Gagrat and Somashekhar Amrith Naimpally, Proximity approach to

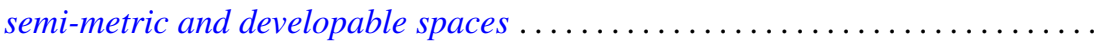

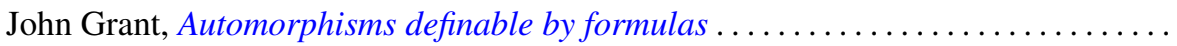

Walter Kurt Hayman, Differential inequalities and local valency ..............

Wolfgang H. Heil, Testing 3-manifolds for projective planes . . . . . . . . . . . . .

107

Melvin Hochster and Louis Jackson Ratliff, Jr., Five theorems on Macaulay

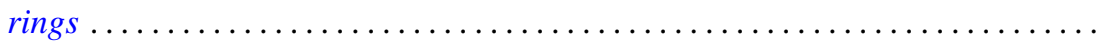

Thomas Benton Hoover, Operator algebras with reducing invariant subspaces ....

James Edgar Keesling, Topological groups whose underlying spaces are separable

Fréchet manifolds...

Frank Leroy Knowles, Idempotents in the boundary of a Lie group . .

191

George Edward Lang, The evaluation map and EHP sequences ...

201

Everette Lee May, Jr, Localizing the spectrum . . . . . . . . . . . .

211

Frank Belsley Miles, Existence of special $K$-sets in certain locally compact abelian groups.

Susan Montgomery, A generalization of a theorem of Jacobson. II . .

T. S. Motzkin and J. L. Walsh, Equilibrium of inverse-distance forces in

three-dimensions.

Arunava Mukherjea and Nicolas A. Tserpes, Invariant measures and the converse

of Haar's theorem on semitopological semigroups .

James Waring Noonan, On close-to-convex functions of order $\beta$

Donald Steven Passman, The Jacobian of a growth transformation

Dean Blackburn Priest, A mean Stieltjes type integral ........ .

Joe Bill Rhodes, Decomposition of semilattices with applications to topological

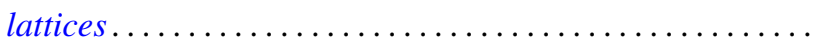

Claus M. Ringel, Socle conditions for $\mathrm{QF}-1$ rings ..........

Richard Rochberg, Linear maps of the disk algebra

Roy W. Ryden, Groups of arithmetic functions under Dirichlet convolution . .

Michael J. Sharpe, A class of operators on excessive functions

Erling Stormer, Automorphisms and equivalence in von Neumann algebras ..

Philip C. Tonne, Matrix representations for linear transformations on series

analytic in the unit disc. 